\title{
La memoria en la evaluación del aprendizaje en ciencias sociales
}

Memory in the evaluation of learning in social sciences

\author{
Blanca C. Bolaños Retavisca \\ Secretaria de educación de Funza, Colombia \\ bc.bolanos@uniandes.edu.co
}

Diego F. Mendoza Torres

Secretaria de educación de Bogotá, Colombia

df.mendoza@uniandes.edu.co

\section{Raúl Vásquez Sánchez}

Secretaria de educación de Funza, Colombia

r.vasquez@uniandes.edu.co

Yenda F. Jaramillo Cano

Secretaria de educación de Cundinamarca, Colombia

yf.jaramillo@uniandes.edu.co

\section{ReSUMEN:}

En este artículo se presentan los resultados de un estudio que buscó identificar las concepciones de dos docentes de ciencias sociales de una institución educativa oficial de la ciudad de Bogotá, acerca del papel que juega la memoria en los procesos de enseñanza y evaluación del aprendizaje en el aula. Para ello, se empleó un enfoque cualitativo, se realizaron entrevistas semiestructuradas, observaciones de prácticas pedagógicas y análisis de resultados de desempeño en una prueba estandarizada sobre civilizaciones precolombinas aplicada a estudiantes de grado sexto, siendo esta última un producto adicional del proceso. Se concluyó que para las docentes la memoria cumple un papel relevante en términos de: huella del aprendizaje, base del desarrollo de niveles de pensamiento superior y significación de lo aprendido. No obstante, se observó que no necesariamente dichas concepciones y prácticas inciden en el aprendizaje alcanzado por sus estudiantes en términos de niveles de pensamiento superior.

Palabras clave: Memoria, Evaluación del aprendizaje, Enseñanza de las ciencias sociales, Niveles de pensamiento.

\section{ABstraCT:}

This article presents the results of a study that sought to identify the conceptions of two social science teachers from an official educational institution in the Bogotá city, about the role that memory plays in the processes of teaching and evaluating learning in the classroom. For this, was used a qualitative approach. Semi structured interviews were conducted, observations of pedagogical practices and analysis of performance results in a standardized test on pre-Columbian civilizations applied to sixth grade students, the latter being an additional product of the process. It was concluded that memory plays an important role for teachers in terms of: learning footprint, basis of the development of higher levels of thinking and meaning of what has been learned. However, it was observed that these conceptions and practices do not necessarily affect the learning achieved by their students in terms of higher levels of thinking.

KEYWORDS: Memory, Evaluation of learning, Teaching of social sciences, Levels of thought.

\section{INTRODUCCIÓN}

Reflexionar en torno a la evaluación es importante para aquellos profesionales e investigadores inmersos en el campo de la educación. Este estudio, enmarcado en un proyecto de investigación en el curso de evaluación del aprendizaje de la Maestría en Educación de la Universidad de los Andes, reconoce la preponderancia que se ha dado a la evaluación de programas educativos y a la evaluación para la selección y 
clasificación de personal (Rose, 2015). Sin embargo, en la presente investigación se adoptó el enfoque de la evaluación del aprendizaje por considerarlo más pertinente.

La necesidad de evaluar los aprendizajes se aborda desde distintos niveles, por ejemplo, las pruebas estandarizadas aplicadas a estudiantes de último grado de bachillerato (Saber $11^{\circ}$ ). Éstas relacionan los aprendizajes con la calidad de la educación impartida tal y como lo expresan Duarte, Bos y Moreno (2012) al afirmar que "la calidad de la educación está representada en los aprendizajes de los alumnos y se mide a través de las pruebas saber" (p. 5).

Sin embargo, los procesos de evaluación del aprendizaje pueden explorarse de manera auténtica en el aula, en tanto que muchas de las políticas públicas tienden a basar sus juicios sobre la calidad y el aprendizaje exclusivamente en pruebas masivas que se toman como un retrato instantáneo del aprendizaje, desconociendo la riqueza que ofrece el proceso de evaluación en el aula, real en términos de experiencias vividas por los distintos sujetos que interactúan en las escuelas. Black y William (1998) asumen este reto cuando invitan a introducirse en la llamada "caja negra", esto es, indagar acerca de lo que estudiantes y docentes hacen en el aula, y emplear dicha reflexión para la toma de decisiones, tanto aquellas referidas a la política estatal, como aquellas estrictamente relacionadas con el propio docente y su instrucción. Porque el papel de un maestro eficaz es llegar a la toma de decisiones reflexivas basada en evidencia obtenida a través de la evaluación, el razonamiento y la experiencia (McMillan, 2001).

Por ello, se decidió llevar a cabo el estudio en dos aulas de una institución educativa oficial de Bogotá, la cual, según datos suministrados por docentes y directivos, se caracteriza por presentar tasas considerables de repitencia, especialmente en grado sexto (cerca del 20\%) que estarían relacionadas con las denominadas “malas prácticas de evaluación”. Según Black y William (1998) éstas aparecen cuando los métodos de evaluación utilizados por los maestros no son eficaces en la promoción de un buen aprendizaje, las prácticas de calificación tienden a enfatizar la competencia en lugar de la mejora personal y la retroalimentación en la evaluación (cuando esta tiene lugar) tiene un impacto negativo en algunos estudiantes, en especial en aquellos con bajo desempeño.

Sin embargo, vale la pena aclarar que, para efectos de la presente investigación, no se ha pensado en el docente como un problema, antes bien, en muchos casos más allá del señalamiento a los maestros, la crítica se ha enfocado en el método, que para algunos autores como Montoya (2015) y Santiago, (2005) corresponde a un currículo tradicional, de prácticas memorísticas y mecánicas. Es allí donde se quiere revelar que, de alguna forma, la idea de la memoria en la enseñanza y la evaluación en estos días se observa desde un punto de vista que la señala como innecesaria y obsoleta, precisamente en el escenario de la cibercultura (Rueda, 2012) en el que se privilegia la externalización del saber y el manejo del conocimiento desde dispositivos digitales de acceso a la memoria global.

En el caso de la enseñanza-aprendizaje de las ciencias sociales, la memoria ha tenido un papel relevante y a la vez polémico, ya que es difícil establecer si en la práctica se fomenta un pensamiento declarativo de nivel básico o un pensamiento superior; entendido el primero como un pensamiento memorístico centrado en procesos de recordar y reproducir, mientras el segundo se asume como la capacidad de análisis, síntesis, contextualización, resolución de problemas, etc., (Gómez \& Miralles, 2015).

De hecho, Gómez y Miralles (2015), encontraron en su investigación con estudiantes de básica primaria y secundaria de instituciones educativas de la región de Murcia (España), que la práctica memorística en la evaluación de la historia ha dejado de lado el pensamiento reflexivo, crítico y creativo propio de las capacidades intelectuales superiores tales como análisis, síntesis, conceptualización, manejo de información, pensamiento sistémico, investigación y meta-cognición, para centrarse en la retención de hechos y conceptos estáticos e inconexos.

Por tanto, surgen las siguientes preguntas: ¿cuáles son las concepciones de dos docentes de ciencias sociales de una institución educativa oficial de Bogotá, acerca del papel de la memoria en la enseñanza y 
en la evaluación del aprendizaje en el aula? ¿Cómo dichas concepciones se relacionan con los niveles de pensamiento alcanzados por sus estudiantes de grado sexto (evidenciados en los resultados de una prueba sobre civilizaciones precolombinas)?

Para resolver dicha pregunta, se diseñó un estudio bajo el paradigma hermenéutico que, desde un enfoque cualitativo, permitió identificar y caracterizar las concepciones que dos docentes tenían acerca del papel que juega la memoria en su práctica pedagógica, mediante entrevistas semiestructuradas y observaciones no participantes de clase. Posteriormente, se construyó e implementó un instrumento de evaluación del aprendizaje, que permitiera comprender hasta qué punto los estudiantes dominaron el constructo de civilizaciones precolombinas, y qué niveles de pensamiento fueron alcanzados por ellos tras la unidad desarrollada por las maestras.

En los siguientes apartados se presenta el marco de referencia que fundamenta teóricamente la investigación, se describe la metodología y el diseño del estudio, se ponen a consideración los resultados y la discusión de los principales hallazgos y, finalmente, se exponen las conclusiones derivadas del estudio.

\section{MARCO DE REFERENCIA}

En esta sección se presentan los referentes teóricos que guían la investigación y que apuntan a la comprensión de las principales categorías y conceptos tratados. En primera instancia, se abordan las concepciones docentes como base de la práctica pedagógica, y las perspectivas que desde la literatura han dado lugar a la idea de memoria. Además, se explica el concepto de niveles de pensamiento y el lugar de la memoria desde dicha concepción. En segundo lugar, se explora el campo de la evaluación del aprendizaje y, finalmente, algunos aspectos técnicos en el diseño de pruebas.

\section{Concepciones docentes, memoria, aprendizaje y niveles de pensamiento}

Para Feixas (2010) las concepciones docentes son el conjunto de significados especiales que los profesionales de la educación otorgan a un fenómeno, los cuales están estrechamente relacionados con sus prácticas. De allí que, para este estudio fue fundamental explorar las concepciones docentes sobre la memoria en la práctica pedagógica, puesto que presumimos que los profesores basan su quehacer en el aula y la toma de sus decisiones de planeación, enseñanza y evaluación de acuerdo a las ideas o creencias que adquieren a lo largo de su experiencia profesional.

\footnotetext{
"El pensamiento docente constituye un marco de referencia integrado por un cúmulo de teorías implícitas, representaciones, imágenes, suposiciones, nociones, ideas, intenciones, proyectos, supuestos, hipótesis, creencias, actitudes, intereses y valores que son susceptibles de influir en la selección de criterios para tomar decisiones sobre qué, cuándo y cómo planear, actuar y evaluar los procesos de enseñanza y de aprendizaje". (Coll \& Miras (1993) en Barron 2015, p. 37).
}

A partir de lo anterior, se hace indispensable analizar, desde las concepciones docentes, la manera cómo la memoria, tema central de esta investigación, se hace presente en su quehacer pedagógico en el aula y se traduce en prácticas de enseñanza y evaluación del aprendizaje, teniendo en cuenta que, según los modelos pedagógicos contemporáneos, pensar en la memoria en el ámbito educativo no está bien visto, porque esta concepción asume que la capacidad de memoria se equipara a un método memorístico y a un modelo de educación bancaria (Freire 1968).

No obstante, la memoria se presenta como ese algo que le da sentido y proporción a la vida, la identidad y lo conocido. Cuando se explora el concepto de memoria, conviene distinguir dos grandes escenarios de comprensión. El primero, hace referencia a la concepción de memoria individual o psicológica, asumida como el resultado de procesos que ocurren en la mente de cada persona y que ha dado lugar a dos líneas de investigación: la neuropsicológica y la cognitiva (Ballesteros, 1999). Adicionalmente, está la 
concepción colectiva o sociológica de la memoria, que la comprende como una construcción socio-histórica y políticamente cuestionable, proveedora de significados compartidos que dan lugar a la identidad cultural (Heller, 2001).

Considerando lo anterior, este estudio se enmarca en la categoría de memoria individual, aunque, vale la pena anotar que en el campo de las ciencias sociales el conocimiento que se pone en juego en la práctica pedagógica y disciplinar, en muchos de los casos se materializa como representación social de la memoria colectiva, transmitida a otros mediante el acto educativo.

Así, el proceso que permite adquirir el conocimiento sobre el mundo en el que vivimos es el aprendizaje, mientras que el proceso por el que ese conocimiento es codificado, almacenado, consolidado, y posteriormente recuperado, es la memoria (Aguado, 2001). Dentro del círculo neuronal es imposible distinguir entre el aprendizaje y la memoria, entonces, se consideran procesos cognitivos íntimamente relacionados. Por lo tanto, se asume que lo aprendido está directamente relacionado con la memoria, porque ésta es la expresión de la capacidad que tiene el ser humano para recuperar las informaciones adquiridas (Ortega \& Franco, 2010).

Desde finales del siglo XIX, la memoria se convirtió en tema de estudio de la psicología, de hecho, la clasificación de los tipos de memoria es reciente como lo describe Allen (2012); al comentar que William James (1842 - 1910), fue el pionero en formular la distinción entre los tipos de memoria, pues concluyó que existía una memoria primaria y otra llamada memoria secundaria. Un tiempo después, apareció la denominada teoría multialmacén de Richard Atkinson y Richard Shiffrin (1968) en la cual se entiende que la información va pasando por diferentes almacenes de memoria a medida que se va procesando. Según la teoría de estos dos autores, contamos con tres tipos distintos de memoria: la memoria sensorial, la memoria a corto plazo (MCP) y la memoria a largo plazo (MLP).

A pesar de la interdependencia existente entre memoria y aprendizaje, algunos autores llegan a afirmar que los procesos memorísticos, así como, el recordar y reproducir, hacen parte de los llamados niveles de pensamiento inferior, presentes en algunas taxonomías (Webb, 1997). En este sentido, cuando se hace referencia a niveles de pensamiento, es pertinente citar algunos conceptos orientados desde el constructivismo. Desde esta perspectiva, las habilidades del pensamiento, entendidas como operaciones mentales, "son el conjunto de acciones interiorizadas, organizadas y coordinadas en función de las cuales realizamos la elaboración de la información que recibimos" (Márquez, 1998). Por otra parte, Lipman (1991) define niveles de pensamiento como

"El conjunto de acciones interiorizadas, organizadas y coordinadas, que propicien un adecuado procesamiento de la información, enfocadas tanto a la información a procesar en sí, como también a las estructuras, procesos y estrategias que están siendo empleadas al procesarla” (citado en Parga, 2002, p. 10).

Ahora bien, Nickerson (1990) desarrolla dos ideas, la primera enfocada a las habilidades de pensamiento de bajo orden, indicando que son aquellas que corresponden a una práctica muy específica, por ejemplo, identificar; la segunda idea se relaciona con las habilidades de pensamiento superior o de alto orden, en las cuales se requiere de la combinación y uso pertinente y oportuno de habilidades de pensamiento de bajo orden, como ejemplo es preciso nombrar el razonamiento (hipotético, analógico, etc.) y la solución de problemas.

Finalmente, Costa (1994) define el pensamiento de orden superior como:

"la habilidad que tenemos para planear una estrategia que nos permita obtener la información que necesitamos. También nos permite estar conscientes de nuestros pasos y estrategias durante el proceso de solución de problemas y de evaluar la productividad de nuestro propio pensamiento" (citado en Parga, 2002, p. 10). 


\section{La evaluación del aprendizaje y el diseño de pruebas}

En el estudio “¿Qué pensamos cuando evaluamos? La evaluación al tablero” de Herrera, Gámez, Torres, Corredor \& Quintero (2008), los autores se basaron en el "hacer y decir" de los docentes actores del proyecto, encontrando que para los maestros participantes la calificación es una prioridad convirtiéndose "en una medida del conocimiento adquirido por los estudiantes, además de ser requisito exigido institucionalmente" (p. 41). Este estudio afirma que en las prácticas evaluativas predomina el paradigma conductual en los docentes, y aunque ellos manifiestan que su evaluación está centrada en los procesos, los estudiantes declaran que no conocen los parámetros que se tienen en cuenta en la evaluación, se concluye, pues, que no existe coherencia entre el decir y el hacer del docente.

Por otra parte, en el trabajo de Puentes (2009) titulado "Tensiones y distensiones en la práctica evaluativa", los investigadores concluyeron que los docentes evalúan como ellos fueron evaluados, creando concepciones. Observaron también que los docentes tienen diferentes interpretaciones con relación a la propuesta de evaluación contemplada en el programa, esto conlleva a que se implemente de varias formas y que existan diferentes tensiones y distensiones entre ellas. Cabe la pena resaltar que los docentes conciben su forma de evaluar de manera cualitativa, formativa y constructiva, mientras que los estudiantes hacen énfasis en lo cuantitativo y sumativo, mostrándose preocupados porque perciben la evaluación como una estrategia para ser medidos.

Aunque algunos autores advierten que una sola prueba no es suficiente para dar cuenta del aprendizaje (Brookhart \& Nitko, 2007), creemos que sí puede ser útil para la medición de constructos trabajados por los docentes, bajo criterios de fiabilidad y validez (Reynolds, Livingston \& Wilson, 2006). Al respecto, observamos el aporte de Downing (2006) que propone el "manual de desarrollo de pruebas", compuesto por doce pasos que resultan ser útiles en la construcción y organización de los instrumentos de evaluación. Cada uno de estos pasos requiere de una técnica específica dependiendo del tipo de prueba, de sus destinatarios, de los recursos disponibles y de la capacitación de los desarrolladores.

Los pasos propuestos por Downing (2006) "proporcionan un marco de organización conveniente para la recopilación y notificación de todas las fuentes de evidencia de validez para un programa de pruebas” ( $p$. 4). Para ello, es indispensable conocer las tareas que garantizan el éxito en el desarrollo de la prueba, a saber: elaboración del plan general, definición de contenidos, especificaciones de la prueba, desarrollo de artículos, diseño y montaje de la prueba, producción de prueba, administración de la prueba, respuestas de la prueba, puntajes de aprobación, informe de los resultados de la prueba, banca de artículos e informe técnico de la prueba.

También, durante la construcción de la prueba es importante tener especificaciones que permiten describir la evaluación y tienen en cuenta los siguientes componentes propuestos por López (2013): "descripción del contexto, propósito de la evaluación; definir un constructo, describir las actividades, describir lo que se espera de los estudiantes y describir de qué manera se va a calificar" (p. 106). Estos elementos contribuyeron a desarrollar una planeación acertada y eficaz del instrumento de evaluación que fue diseñado en esta investigación.

Además, al planear un instrumento se debe tener en cuenta una tabla de especificaciones que permite dar respuesta a las siguientes preguntas propuestas por López (2013):

“¿Para qué evaluar? ¿En dónde voy a evaluar? ¿A quién voy a evaluar? ¿Qué voy a evaluar? ¿Cómo lo voy a evaluar? ¿Cómo voy a calificar los desempeños de mis estudiantes? ¿Cómo voy a interpretar los resultados de la evaluación?” (p. 106).

Esta información le facilita al docente la tarea de diseñar sus evaluaciones para un contexto específico garantizando la obtención de resultados de mayor confiabilidad.

Reynolds y sus colegas (2006), también proponen desarrollar una tabla de especificaciones y utilizarla para garantizar la coherencia entre los constructos trabajados en clase y la temática de la prueba. Esta tabla presenta 
las principales áreas y contenidos que serán tratados y que surgen de los objetivos educativos que se incluirán en dicha prueba. Los objetivos que orientan la actividad evaluativa parten de los niveles de la taxonomía cognitiva, los cuales, según Reynolds, Livingston \& Wilson (2006), “permiten considerar la complejidad de los procesos cognitivos que se quiere medir" (p. 173). De acuerdo con este autor, incluir los procesos cognitivos en la prueba garantiza que los docentes tengan una mayor gama de elementos que pueden ser incorporados en su instrumento de evaluación.

De acuerdo con lo anterior, se propone tener en cuenta la taxonomía de Webb (1997), esta establece cuatro niveles de pensamiento: recordar y reproducir, habilidades y conceptos, pensamiento estratégico y pensamiento extendido. Cada uno de ellos establece diferentes verbos que permiten identificar qué logra hacer el estudiante con el conocimiento adquirido.

Finalmente, al culminar el proceso de diseño y aplicación del instrumento evaluativo, se recomienda brindar una retroalimentación efectiva a los estudiantes que refleje, no sólo su dominio de temas, sino también el nivel alcanzado de acuerdo a la taxonomía de Webb. En este sentido, dicha retroalimentación debe ser incluida dentro del proceso como un elemento positivo que favorezca en los estudiantes la adquisición de un aprendizaje significativo y que lo motive con el fin de tomar acciones correctivas sobre su desempeño encaminadas hacia el mejoramiento (McMillan, 2007).

\section{Metodología}

Este estudio se enmarca en el paradigma histórico hermenéutico propio de la investigación cualitativa. Esto, debido al interés por comprender el papel que juega la memoria en la evaluación del aprendizaje de las ciencias sociales en estudiantes y docentes de grado sexto de un colegio oficial de Bogotá.

En este sentido, este enfoque muestra interés natural por dar cuenta de la acción humana y su interrelación con el medio social (Gutiérrez, 2004). Es por ello que, obedeciendo a los supuestos epistemológicos del mismo, se plantea la necesidad de una práctica metodológica de carácter cualitativo, principalmente por su fecunda articulación con las dimensiones histórica, cultural, socio-política, y contextual (Lepe, 2009). Adicionalmente, para Denzin y Lincoln (2000), la investigación cualitativa sitúa al observador en el mundo, utilizando una serie de prácticas interpretativas que lo hacen visible. Además, estas prácticas transforman el mundo, debido a que lo plasman en una serie de representaciones textuales a partir de los datos recogidos en el campo mediante observaciones, entrevistas, conversaciones o fotografías.

Las técnicas de recolección de información usadas corresponden a dos observaciones no participantes de prácticas en el aula y una entrevista semiestructurada por cada docente. En relación a la primera, se dice que en esta el docente investigador se limita a registrar la información que aparece ante él en diarios de campo, cuidando de no interactuar ni interferir con el objeto de estudio (Flick, 2007), por tanto, en esta técnica el investigador se mantiene al margen del fenómeno objeto de estudio. En segundo lugar, la entrevista semiestructurada gira en torno a una temática propuesta por el investigador mediante un diálogo establecido con la finalidad de conocer el punto de vista o la experiencia del entrevistado (Stake, 2010).

En el presente estudio se contó con la colaboración de dos docentes del área de ciencias sociales de un colegio oficial de Bogotá, uno de la jornada mañana y otro de la jornada tarde, en adelante D1 y D2. Del mismo modo, participaron 55 estudiantes de grado sexto distribuidos en dos grupos, uno por cada jornada. Todos ellos fueron informados, mediante reuniones, del propósito de la investigación y se les solicitó su participación libre y voluntaria. Además, teniendo en cuenta que parte de la muestra corresponde a jóvenes menores de edad, se pidió autorización a los adultos responsables de cada uno de ellos para su participación. Para ello, se envió el documento denominado consentimiento informado para mayores a cargo de menores de edad. En él, se explican los pormenores de la investigación y se suministra información suficiente para contactar a los docentes investigadores en caso de cualquier inquietud. Del mismo modo, los docentes fueron 
informados sobre el propósito del estudio y se les solicitó también su participación voluntaria. Para ello, se diseñó y aplicó el documento denominado consentimiento informado para docentes participantes.

El diseño del estudio contempló dos fases. En la primera, se identificaron y caracterizaron las concepciones docentes sobre el papel de la memoria en la enseñanza y en la evaluación del aprendizaje de las ciencias sociales, tras la implementación de los instrumentos que proporcionaron datos importantes e información espontánea y valiosa que fue codificada y posteriormente organizada en subcategorías y categorías que permitieron, tal como lo manifiesta Flick (2007), la triangulación de datos y su correspondiente análisis. Durante la segunda fase de la investigación, se diseñó e implementó un instrumento totalmente validado para la evaluación del aprendizaje, siguiendo las recomendaciones de Downing (2006), López (2013) y Reynolds y otros (2006), tal como se describe con detalle en el siguiente apartado.

\section{Diseño y validación de la prueba}

La prueba diseñada toma como marco de referencia los presupuestos establecidos en las temáticas de los ejes generadores que aparecen en los lineamientos curriculares en ciencias sociales (MEN, 2002). El sexto eje, por ejemplo, hace referencia a la identidad y la memoria colectiva, involucrando disciplinas como historia, antropología, geografía, entre otras. En cuanto a los estándares (MEN, 2004) asumimos la coherencia de contenidos en ciencias sociales, que para el grado sexto y séptimo plantea el reconocimiento de redes complejas de relaciones entre eventos históricos, sus causas, sus consecuencias y su incidencia en la vida de los diferentes agentes involucrados. Además, se busca que los estudiantes en estos grados establezcan correlaciones entre las culturas y sus épocas, comparen sistemas políticos, localicen culturas en espacios geográficos, comparen las organizaciones económicas de diferentes culturas y propongan explicaciones para las semejanzas y diferencias que encuentran.

En consideración a lo anterior, los docentes investigadores decidimos establecer un diálogo con las docentes participantes para la definición de los componentes que serían incluidos en la prueba, dando lugar a la tabla de especificaciones que se presenta a continuación (Tabla 1), elaborada según las recomendaciones de López (2013). 


\section{TABLA 1}

\begin{tabular}{|c|c|}
\hline & Tabla de especificaciones \\
\hline Objetivo de la evaluación & $\begin{array}{l}\text { El estudiante comprende las caracteristicas básicas de las principales civilizaciones precolombinas } \\
\text { de América. }\end{array}$ \\
\hline $\begin{array}{l}\text { Caracteristicas del } \\
\text { contexto }\end{array}$ & $\begin{array}{l}\text { La prueba se realiza con estudiantes del Colegio Distrital Silveria Espinoza de Rendón, de dos } \\
\text { grupos de grado sexto ( } 603 \mathrm{JM} \text { y } 601 \mathrm{JT} \text { ) con edades comprendidas entre los } 11 \text { y los } 13 \text { años, } \\
\text { pertenecientes a estratos socioeconómicos } 2 \text { y } 3 \text {. Hay en promedio } 27 \text { estudiantes por sula, } \\
\text { aproximadamente } 55 \% \text { nirias y } 45 \% \text { niños, y en cada grupo hay un estudiante con necesidades } \\
\text { educativas especiales leves ya que la institución integra a esta población. Se contará con la } \\
\text { participación de dos docentes, una de la jomada mañana y una de la jomada tarde. }\end{array}$ \\
\hline $\begin{array}{l}\text { Caracteristicas de los } \\
\text { estudiantes }\end{array}$ & $\begin{array}{l}\text { Los estudiantes que serán evaluados mediante el instrumento se caracterizan por tener } \\
\text { intereses ligados a las actividades deportivas tales como el futbol y el voleibol; artísticas como el } \\
\text { teatro, la música y el baile, preferencias reflejadas en un festival de talentos celebrado cada aìo. } \\
\text { Durante las clases, expresan motivación e interés por el uso del juego, la proyección de películas, } \\
\text { imágenes y otras actividades interactivas que involucran el empleo del computador, smart tv, } \\
\text { tabletas, etc. Son chicos curiosos, preguntones y gustan de la exploración en la web acerca de } \\
\text { misterios y enigmas de la historia. Adicionalmente, los grupos son heterogéneos en términos de } \\
\text { rendimiento académico, encontrándose estudiantes con desempeîos superior, slto, acaptable y bajo. }\end{array}$ \\
\hline Area/Contenido & Ciencias Sociales/Civilizaciones precolombinas de América \\
\hline $\begin{array}{l}\text { Objetivo de sprendizaje } \\
\text { que evalús }\end{array}$ & $\begin{array}{l}\text { Esta prueba tiene como objetivo evaluar conocimientos sobre las civilizaciones precolombinas de } \\
\text { América en estudiantes de grado sexto. }\end{array}$ \\
\hline Constructo & Conocimiento sobre las civilizaciones precolombinas en América \\
\hline Tipo de conocimiento & Conocimiento declarativo y procedimental \\
\hline Formato/Tipo de pregunta & 18 preguntas de selección múltiple \\
\hline $\begin{array}{l}\text { Descripción del } \\
\text { procedimiento }\end{array}$ & $\begin{array}{l}\text { Tiempo total: } 45 \text { minutos } \\
\text { papel y lápiz }\end{array}$ \\
\hline Instrucciones & $\begin{array}{l}\text { En el desarrollo de la pruebe, el estudiante, debe leer atentamente cada pregunta y marca la } \\
\text { respuesta en la hoja anexa suministrada para tal fin. El tiempo propuesto para resolverla es máximo } \\
45 \text { minutos. }\end{array}$ \\
\hline Desempeño esperado & $\begin{array}{l}\text { Teniendo en cuenta el propósito de la prueba, se espera obtener evidencias, con las respuestas de } \\
\text { los estudiantes, si el conocimiento es declarativo, debido al uso preponderante de la memoria } \\
\text { durante la enseñanza del constructo. Sin embargo, también se pretende observar el desarrollo de } \\
\text { niveles de pensamiento mas complejos, tales como, los niveles de pensamiento estratégico o } \\
\text { extendido. }\end{array}$ \\
\hline Forma de calificar & $\begin{array}{l}\text { En la prueba se presentan actividades para cada sub constructo. Cada una de estas, se encuentra } \\
\text { relacionada con los niveles de pensamiento propuestos por Webb (Maria Isabel Ramirez, referencia } \\
\text { en clase, agosto } 31 \text { de 2016). Todo esto, para obtener información sobre el nivel alcanzado en cada } \\
\text { uno de los subconstructos, y que nos permitan dar cuenta del tipo de pensamiento declarativo o } \\
\text { procedimental alcanzado por los estudiantes. Adicionalmente, proponemos sistematizar la } \\
\text { información. Para ello, usaremos una matriz en Excel acondicionada para obtener la información } \\
\text { respecto a cada uno de los niveles de pensamiento (ver anexo 4). }\end{array}$ \\
\hline
\end{tabular}

\section{Validación de la prueba}

Las tres estrategias de validación aplicadas durante esta investigación fueron:

\section{A. Validación por expertos}

Se decidió realizar una validación de contenido y constructo por dos expertos en la temática de civilizaciones precolombinas en América, Se esbozó un instrumento de validación para que dos docentes de otra institución revisaran los contenidos, diseño y pertinencia de cada una de las preguntas y opciones de respuesta, así como de la prueba en general escribiendo sus percepciones sobre cada ítem. Esta validación se realizó para verificar el contenido, el constructo, la claridad de las instrucciones y la dificultad de cada pregunta. 


\section{B. Protocolo de pensamiento en voz alta (thinking out loud)}

Se realizó el protocolo de pensando en voz alta, (Li y Shavelson, 2003) Cuyo propósito fue conocer las percepciones de dos participantes voluntarios, con características similares a las de los estudiantes a quienes se aplicaría la prueba de aprendizaje, además de identificar aspectos problemáticos de cada uno de los ítems. Simultáneamente se registraron los comentarios, ideas y sugerencias de los participantes con relación a cada ítem y se transcribieron, para posteriormente ser tenidos en cuenta a la hora de afinar la prueba.

\section{Validación por consecuencia}

Esta estrategia consiste en entrevistar o encuestar a los estudiantes después de la aplicación de una prueba con el fin de obtener información sobre el impacto generado por esta (López 2013). Fue aplicado a los dos grupos de estudiantes de grado sexto finalizando la implementación de la misma.

\section{Resultados y discusión}

A continuación, se presentan los principales hallazgos relacionados con los objetivos de la investigación. En primer lugar, se muestran los resultados alcanzados por los estudiantes en cuanto a las dos categorías evaluadas por la prueba, y calificados de acuerdo a la información de las tablas 2 y 3 . Cabe aclarar que las docentes solicitaron una calificación individual de la prueba, la cual fue alineada con el sistema institucional de evaluación (SIE), y se entregó a cada estudiante el correspondiente informe de retroalimentación. Los resultados individuales se agregaron y analizaron y se presentan en las gráficas 1 a 6 .

TABLA 2

Resultados de cada grupo por nivel de pensamiento según la taxonomía de Webb

\begin{tabular}{|c|c|c|c|c|c|c|c|c|c|c|c|c|c|c|c|c|c|c|}
\hline Pregunta & 1 & 2 & 3 & 4 & 5 & 6 & 7 & 8 & 9 & 10 & 11 & 12 & 13 & 14 & 15 & 16 & 17 & 18 \\
\hline Puntos & 4 & 4 & 4 & 4 & 4 & 4 & 4 & 4 & 4 & 4 & 6 & 6 & 6 & 6 & 6 & 6 & 6 & 18 \\
\hline Nivel de pensamiento & \multicolumn{5}{|c|}{$\begin{array}{l}\text { Recordar y } \\
\text { reproducir }\end{array}$} & \multicolumn{5}{|c|}{$\begin{array}{l}\text { 2. Habilidades y } \\
\text { conceptos }\end{array}$} & \multicolumn{5}{|c|}{$\begin{array}{l}\text { 3. Pensamiento } \\
\text { estratégico }\end{array}$} & \multicolumn{3}{|c|}{$\begin{array}{l}\text { 4. Pensamiento } \\
\text { extendido }\end{array}$} \\
\hline $\begin{array}{l}\text { Obtiene un nivel de } \\
\text { pensamiento } \\
\text { superior }\end{array}$ & \multicolumn{5}{|c|}{20 puntos } & \multicolumn{5}{|c|}{20 puntos } & \multicolumn{5}{|c|}{30 puntos } & \multicolumn{3}{|c|}{30 puntos } \\
\hline $\begin{array}{l}\text { Obtiene un nivel de } \\
\text { pensamiento alto }\end{array}$ & \multicolumn{5}{|c|}{16 puntos } & \multicolumn{5}{|c|}{16 puntos } & \multicolumn{5}{|c|}{24 puntos } & \multicolumn{3}{|c|}{24 puntos } \\
\hline $\begin{array}{l}\text { Obtiene un nivel de } \\
\text { pensamiento aceptable }\end{array}$ & \multicolumn{5}{|c|}{12 puntos } & \multicolumn{5}{|c|}{12 puntos } & \multicolumn{5}{|c|}{18 puntos } & \multicolumn{3}{|c|}{18 puntos } \\
\hline $\begin{array}{l}\text { Obtiene un nivel de } \\
\text { pensamiento bajo }\end{array}$ & \multicolumn{5}{|c|}{8 puntos } & \multicolumn{5}{|c|}{8 puntos } & \multicolumn{5}{|c|}{12 puntos } & \multicolumn{3}{|c|}{12 puntos } \\
\hline $\begin{array}{l}\text { Obtiene un nivel de } \\
\text { pensamiento } \\
\text { insuficiente }\end{array}$ & \multicolumn{5}{|c|}{4 puntos } & \multicolumn{5}{|c|}{4 puntos } & \multicolumn{5}{|c|}{6 puntos } & \multicolumn{3}{|c|}{6 puntos } \\
\hline $\begin{array}{l}\text { No obtiene registro en } \\
\text { el nivel de pensamiento }\end{array}$ & \multicolumn{5}{|c|}{0 puntos } & \multicolumn{5}{|c|}{0 puntos } & \multicolumn{5}{|c|}{0 puntos } & \multicolumn{3}{|c|}{0 puntos } \\
\hline
\end{tabular}


TABLA 3

Ámbitos del dominio del subconstructo

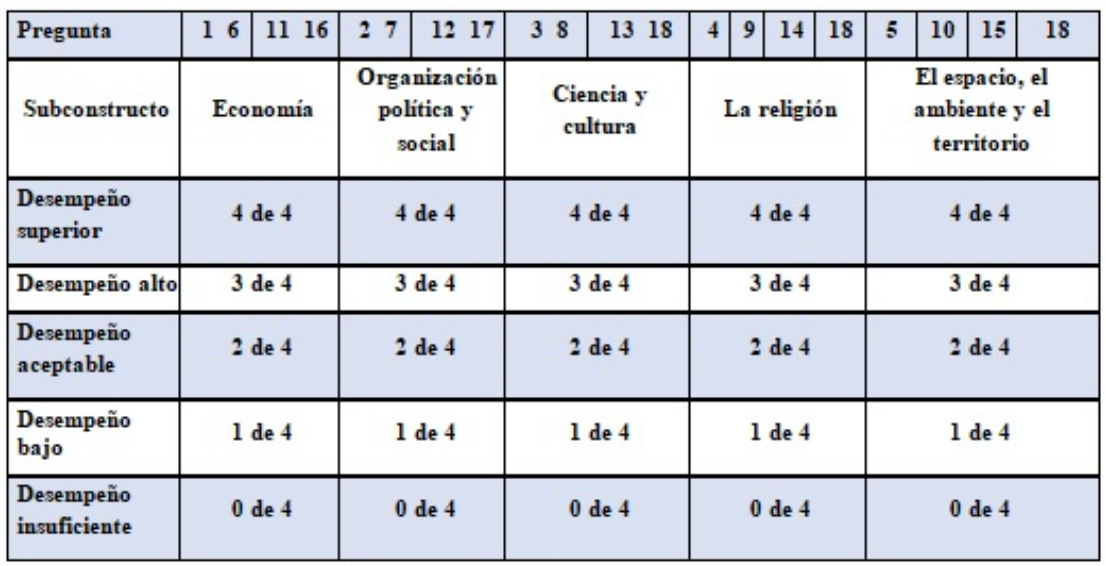

GRÁFICA 1

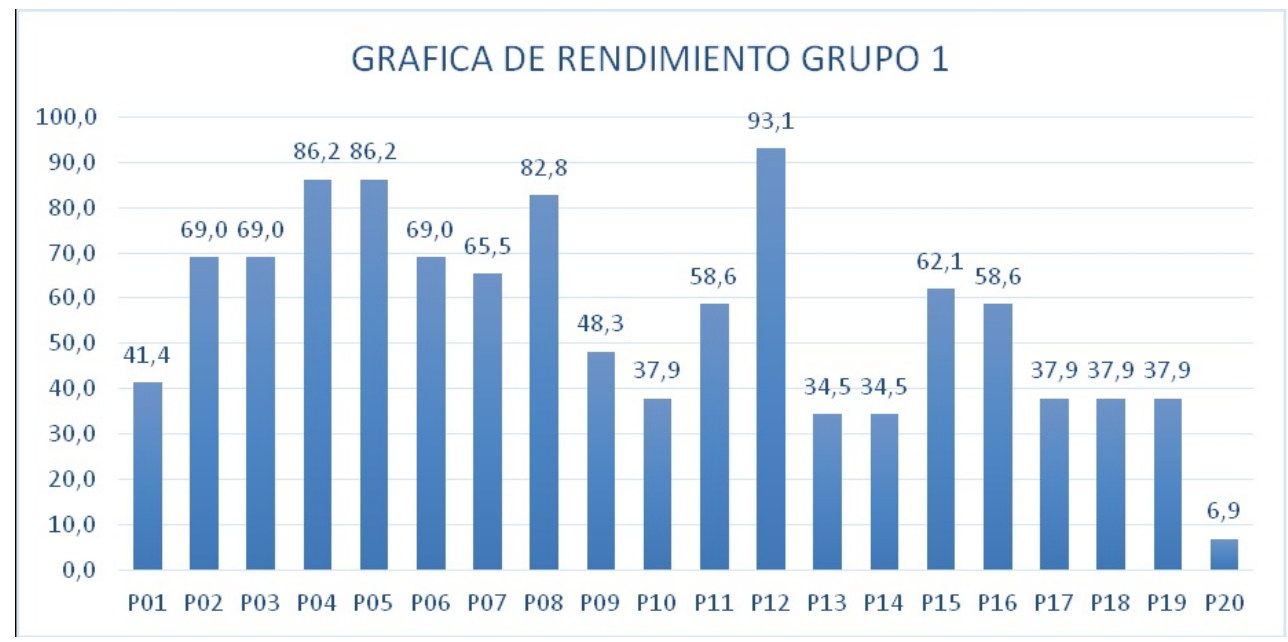

GRÁFICA 2

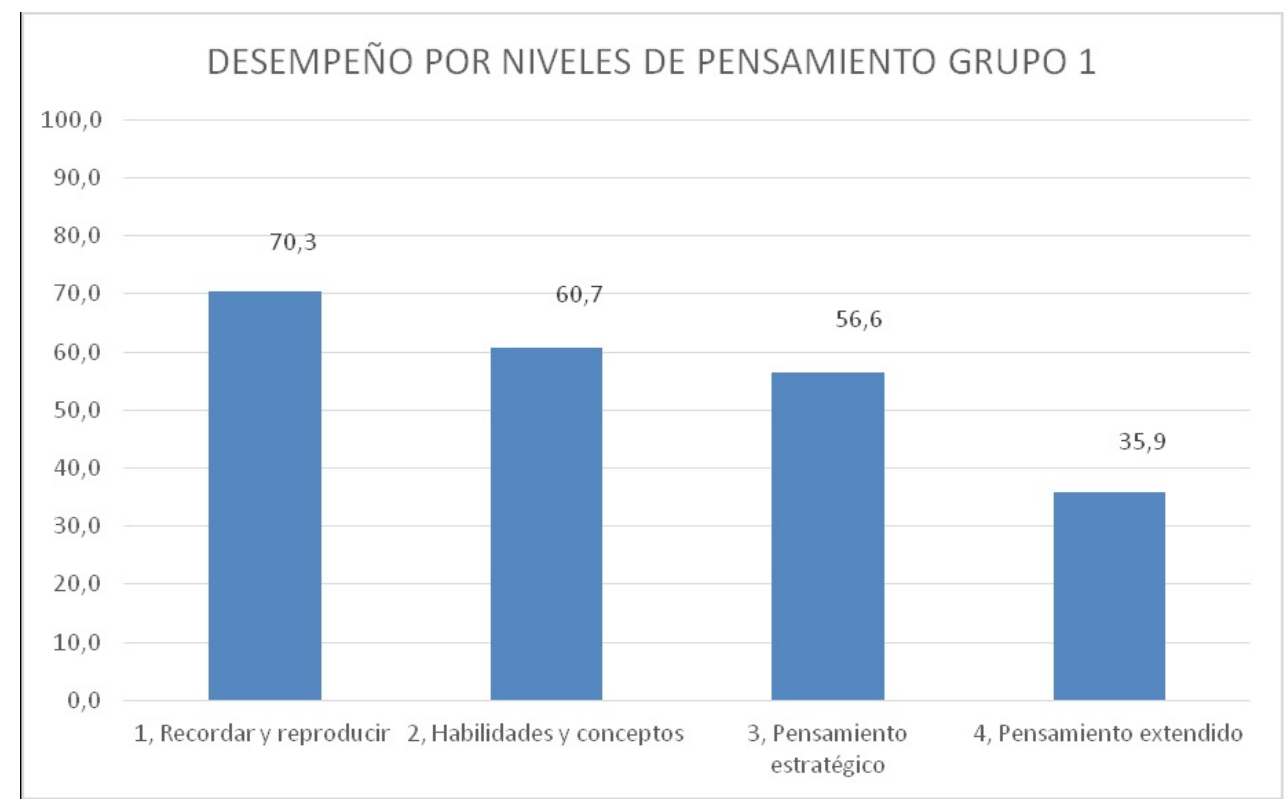


GRÁFICA 3

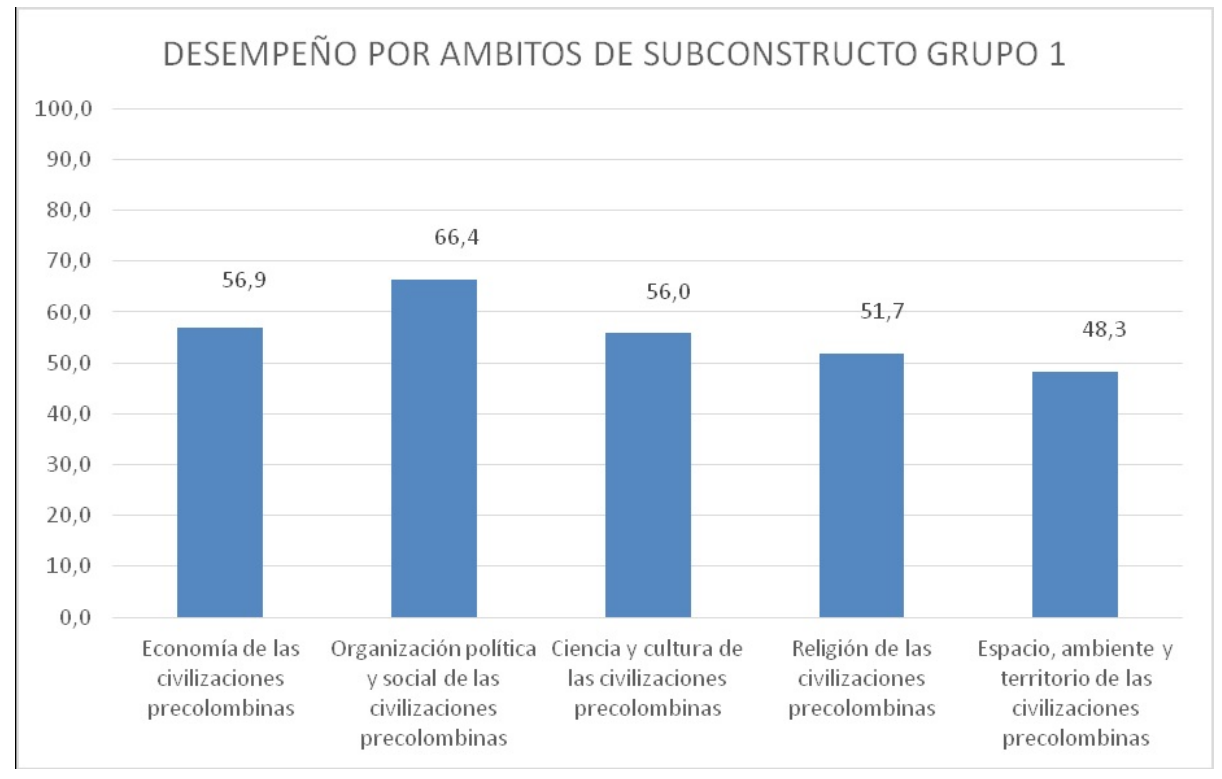

GRÁFICA 4

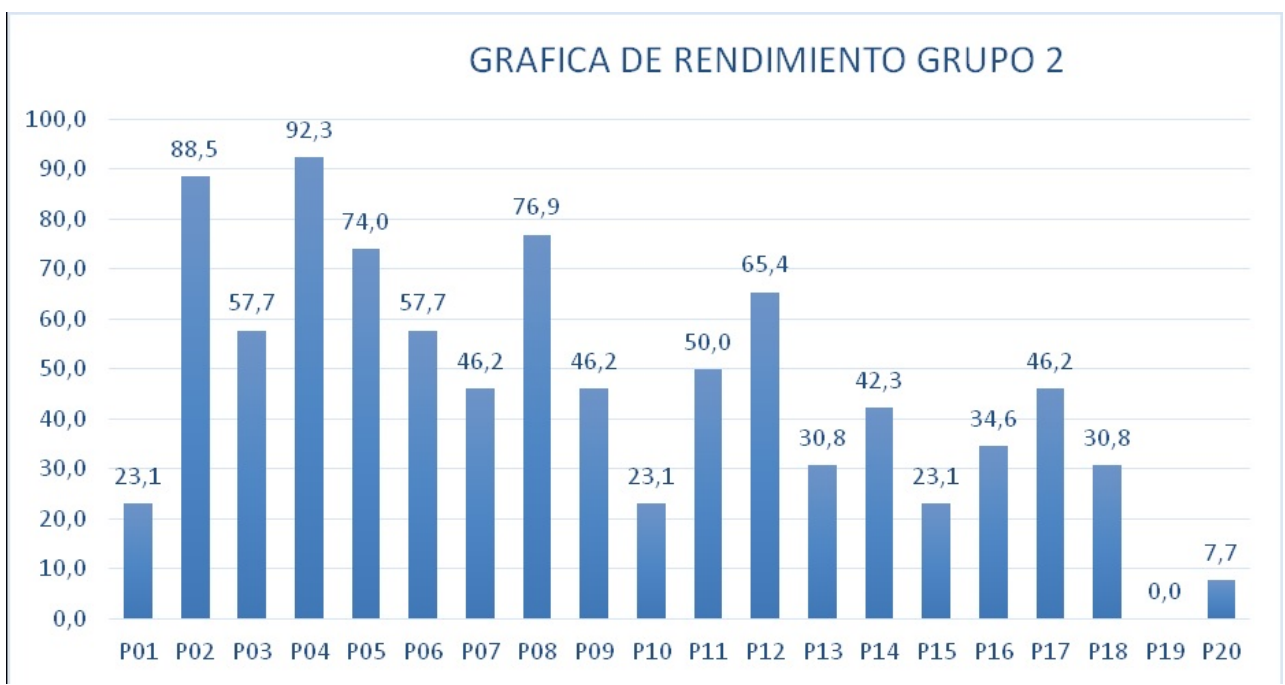

GRÁFICA 5

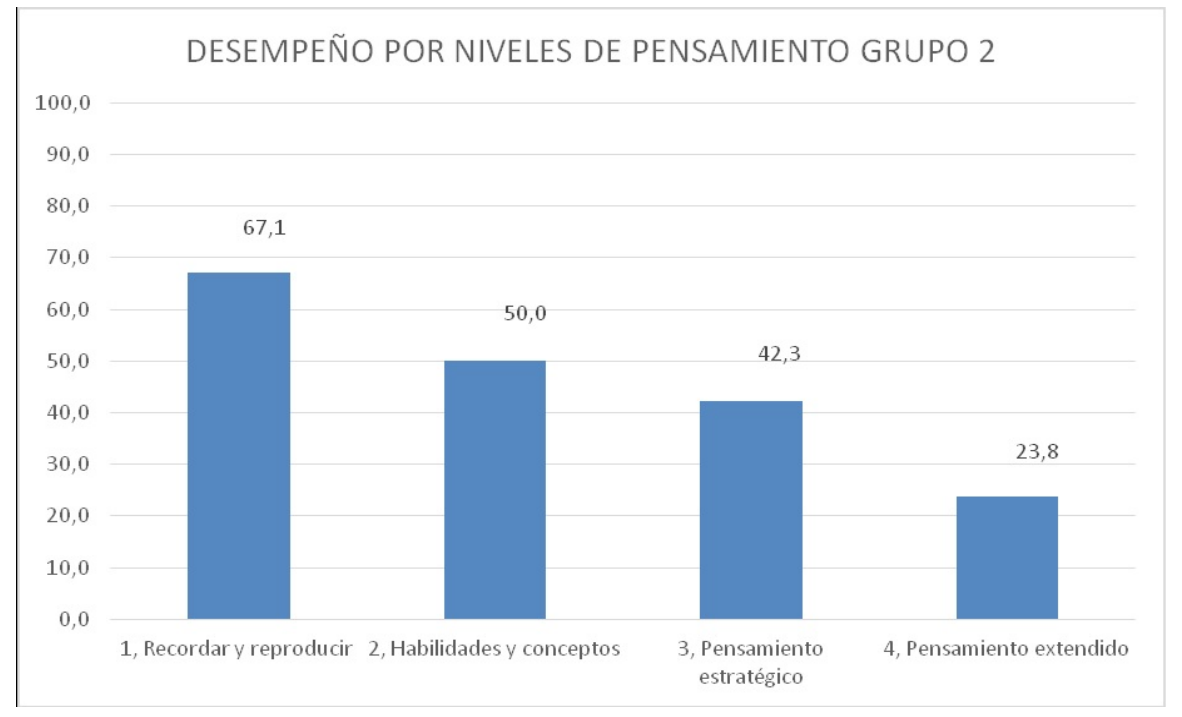




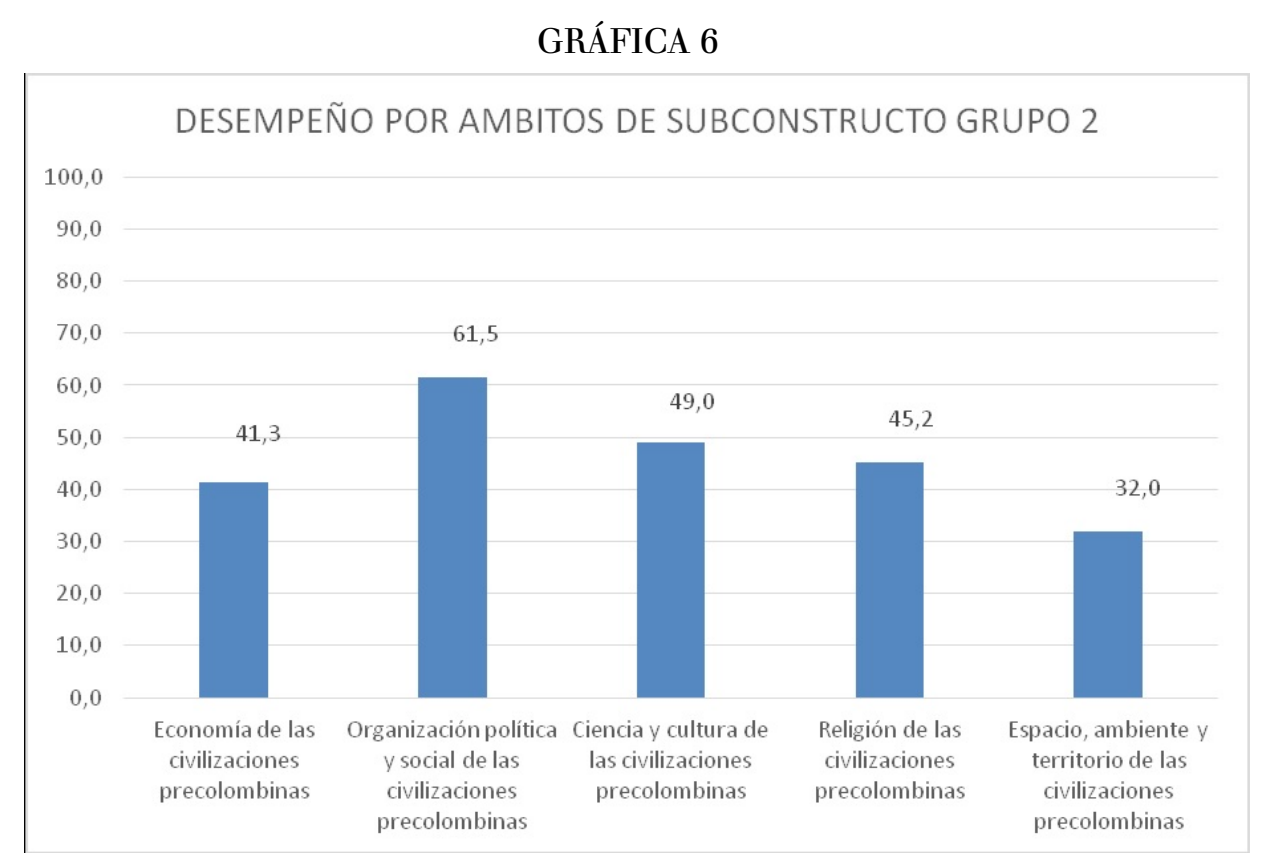

Si observamos el desempeño obtenido por los grupos en cada uno de los ítems de la prueba, es evidente que, según el promedio de aciertos, ninguno de los dos aprobó, teniendo en cuenta que, según el Sistema Institucional de Evaluación, la aprobación se produce cuando se supera el 6,0.

Ahora bien, si consideramos los resultados por niveles de pensamiento, se evidencia que en ambos casos se superan ampliamente las expectativas en los niveles 1 y 2 en los cuales revelan una correspondencia entre las concepciones, las prácticas y los aprendizajes de los estudiantes. Mientras que, en los niveles de pensamiento de orden superior, el rendimiento es más bajo.

Por último, los resultados referentes al dominio de los ámbitos de subconstructo muestran un alto desempeño en el dominio de la organización social y política de las civilizaciones precolombinas, mientras que el ámbito de espacio, ambiente y territorio deben fortalecerse por parte de los docentes con actividades que generen una memoria más significativa durante la etapa de enseñanza.

CONCEPCIONES SOBRE EL PAPEL DE LA MEMORIA EN LA ENSEÑANZA Y EVALUACIÓN DEL APRENDIZAJE DE LAS CIENCIAS SOCIALES

Se presentan a continuación tres concepciones que, tras la implementación de entrevistas semiestructuradas y los datos obtenidos de la observación no participante de las clases de dos docentes, se constituyen en una síntesis de la manera en la que ellas han otorgado un papel a la memoria dentro de las prácticas de aula, el cual se ve reflejado en el tipo de aprendizaje que buscan desarrollar en sus estudiantes, y en la manera como lo evalúan. Estas tres concepciones son: la memoria como huella del aprendizaje, la memoria como base para el desarrollo de niveles de pensamiento superior y memoria significativa.

\section{La memoria como huella del aprendizaje}

Ballesteros (1999) y Aguado (2001) relacionan la memoria con el campo de la psicología y le asignan un carácter individual. De esta manera, es posible afirmar que la memoria, especialmente la memoria semántica o de largo plazo (Allen 2012), se constituye en una huella perdurable de lo aprendido, tanto para el docente como para los estudiantes. 
El docente de ciencias sociales es un poseedor de un saber disciplinar, lo cual se manifiesta a manera de dominio de contenidos, significados, procesos y temáticas en el momento de la instrucción (diario del observador, octubre de 2016). En este sentido, se evidenció que en ningún momento las docentes emplearon libros de texto o herramientas digitales, privilegiando su memoria como la huella del saber en su área. Incluso, las docentes vinculan a su práctica las experiencias que se han convertido en una huella de su memoria individual. Por ejemplo, durante la sesión 2 , cuando se refería a la organización política y social de las culturas indígenas, basada en un orden teocrático, D2 definió de memoria este concepto a sus estudiantes.

Otra cuestión relevante es la huella del aprendizaje retomada como recuerdo antes del inicio de una clase, lo cual le permite al docente evaluar la eficacia en la enseñanza de los temas vistos y darle una secuencia al desarrollo de su práctica. Por ejemplo, D1 expresa que:

"la primera parte es como el recorderis de la clase anterior, entonces chicos recordemos que hicimos la clase pasada, me dan su opinión. Bueno ¿Quién me hace la síntesis de las conclusiones que se sacaron la clase anterior? Entonces, a partir de ello continúo con el siguiente tema, porque la idea es seguir, como hacer una secuencia de lo que se está trabajando a nivel de historia" (Entrevista, noviembre de 2016).

Por lo tanto, se asume que lo aprendido está directamente relacionado con la memoria, porque ésta es la expresión de la capacidad que tiene el ser humano para recuperar las informaciones adquiridas (Ortega \& Franco, 2010), lo cual se evidencia en algunas prácticas de evaluación del aprendizaje en las que las docentes piden a sus estudiantes que recuerden conceptos y los reproduzcan con exactitud (diario del observador, septiembre de 2016). En la sesión 2, D1 felicita a uno de sus estudiantes que corrige a otro compañero cuando afirmaba que los sistemas de agricultura azteca eran los jardines flotantes: “iNo!, interviene una estudiante, se llamaban chinampas y era donde cultivaban los aztecas". D1 dice "imuy bien! Me alegra que lo hayas recordado".

Hemos podido identificar que las actividades de escritura en el desarrollo de las clases, según las profesoras, son importantes para mostrar el aprendizaje de los estudiantes (entrevista, noviembre de 2016). Ya que, como afirma D2, "lo que no se escribe no se recuerda". Es allí donde el cuaderno constituye un dispositivo de soporte y evidencia de la memoria como huella del aprendizaje. En este sentido la docente comenta: "Entonces que bien que yo pueda volver y tomar las notas y decir: joye mira éste escrito lo hice yo, éste dibujo lo hice yo, esto fue lo que yo entendí! porque en el cuaderno se refleja lo que ellos entienden".

En cuanto a los resultados de la prueba (gráficas 3 y 6), hemos de advertir que el desempeño de los estudiantes fue similar en los diferentes ámbitos de subconstructo, sin embargo, la huella del aprendizaje sobre organización política y social sobresale frente a los otros temas. D1, por ejemplo, hizo énfasis en este aspecto durante la evaluación que aplicó a sus estudiantes en la segunda sesión de observación, y dedicó más tiempo a la retroalimentación sobre ítems de este subconstructo (diario del observador, octubre de 2016).

\section{La memoria como base para el desarrollo de niveles de pensamiento superior}

Teniendo en cuenta que las habilidades de pensamiento son el conjunto de acciones interiorizadas, organizadas y coordinadas (Márquez, 1998), las cuales se manifiestan a través de niveles de pensamiento de bajo orden o de pensamiento superior (Nickerson, 1990), se pudo evidenciar, dentro de las concepciones de las docentes, una clara tendencia a identificar la memoria como fundamental para el desarrollo de niveles 
de pensamiento de orden superior. Para ellas, cuando se asocia un evento en la memoria, se le otorga un significado y esto es base para llegar a niveles superiores de pensamiento. Por ejemplo, las docentes estimulan la memoria visual mediante la proyección de videos o actividades de coloreado de mapas o dibujos con el fin de reafirmar conceptos en principio memorísticos mediante actividades. También proponen la elaboración de grafitis que demandan niveles de pensamiento superior, tales como analizar y criticar, propios del nivel de pensamiento extendido de la taxonomía de Webb (1997). Al respecto, D2 dice:

“:Por qué va a ser importante para nosotros firmar la paz? Porque va a ser el bienestar para un futuro teniendo en cuenta todo un pasado, a quién va a afectar... a mí, al estudiante, al de ahorita y sin memoria es difícil que se puedan dar ese tipo de conocimientos" (Entrevista, noviembre de 2016).

$\mathrm{Al}$ respecto, podemos observar que en la prueba se evidencia una clara tendencia de los estudiantes a tener mejores resultados en el primer nivel de pensamiento, recordar y reproducir. No ocurre lo mismo en los resultados obtenidos en los niveles de pensamiento superior o extendido (ver gráficas 2 y 5 ).

Por otro lado, se usa la memoria para recordar y comparar, encontramos evidencia de esto cuando D1 dice:

"Recuerden cuando vimos la parte de las civilizaciones asiáticas, y vimos cómo se desarrollaron y cómo llegaron a ser civilización, así como cuáles fueron sus aportes. No dejemos de lado eso (...) podemos hacer comparaciones, miremos como los de América hacían esto y cómo lo hacían los de Asia, por ejemplo, en Asia hablamos de la rueda, ¿en América también hablamos de la rueda?" (Diario de campo, septiembre 2016).

\section{Memoria significativa}

En esta categoría se produce una síntesis entre la idea de memoria individual (Ballesteros, 1999) y la de memoria colectiva (Heller, 2001). Es decir, que el aprendizaje se significa cuando la memoria primaria (Allen, 2012) da lugar a una experiencia significativa para el estudiante y el conocimiento pasa directamente a la memoria a largo plazo con carga afectiva.

Resulta evidente que el individuo recuerda y se apropia de las experiencias y conocimientos que le resultan significativos, importantes y/o necesarios. Así, D2 afirma:

\footnotetext{
"El recordar es significativo, es lo que yo quiero, yo recuerdo lo que yo quiero. El repetir es para darle gusto a otra persona, por qué uno no se aprende las tablas de multiplicar, porque lo ponen a repetir sin sentido. Mientras que si para ti es significativo por ejemplo un presidente porque fue el que estaba gobernando cuando tu naciste y te vas a interesar, y vas a recordar y vas a ir y mirar, te vas a preocupar por eso, porque le das un sentido a esa memoria... es significativa." (Diario de campo, septiembre de 2016)
}

También se encontró que las docentes refuerzan lo aprendido a través de imágenes, por ejemplo, en la sesión uno D1 manifestó a sus estudiantes: "Si yo hago un dibujo sobre una teoría que estoy trabajando, puedo aprender más. Entonces, los dibujos que ustedes hagan, son muy importantes”. Esto se da porque, al realizar un esquema, dibujo o gráfica, el estudiante le está otorgando su propio significado al conocimiento. Incluso, en los resultados de la prueba (gráficos 1 y 4), los estudiantes alcanzaron en promedio un mejor desempeño en las preguntas relacionadas con imágenes. Por ejemplo, el número 5 el G1 alcanzó un 86,2\% mientras que el G2 obtuvo un 74,0\%, evidenciando que para ellos fue significativa la actividad de ubicación en el mapa propuesto por ambas docentes en los ejercicios de clase.

Adicionalmente, la memoria es también un legado, es decir, al darle el valor a los acontecimientos históricos las docentes generan experiencias significativas en sus estudiantes. Esto se hizo evidente durante la entrevista cuando D1 indicó: "Se dice que el conocer la historia, nos permite no cometer los mismos errores". De este modo, los estudiantes no necesitarían vivir algunas experiencias en carne propia para comprender las posibles consecuencias, es decir, a partir de lo que otros han vivido los jóvenes pueden tomar decisiones informadas.

Es más, durante la primera sesión, D1 captó el interés de los chicos cuando resaltó el valor de la palabra para los incas, en contraposición a la situación de nuestros días (diario del observador, octubre de 2016) ya 
que "cuando hay un aprendizaje real, jamás en la vida se vuelve a olvidar, y si yo adquirí el aprendizaje era porque tenía necesidad de ese conocimiento como tal, y me va a servir para mi vida" (diario del observador, octubre de 2016).

Vale agregar que los ámbitos de subconstructo incluidos en la prueba, eran realmente significativos como memoria en los estudiantes, tal y como se expresa por parte de un estudiante al finalizar una observación: "El estudiante dice que D1 les pidió que construyeran un cuadro de aspectos de las civilizaciones prehispánicas, a lo que el investigador responde "ok... y qué tipo de instrucciones les dio la docente"... el estudiante indica "ella nos dio varios aspectos para que los investigáramos: ubicación, política, economía, religión, la división de la sociedad y la cultura" ¿y qué hacen con cada uno de esos aspectos?... el estudiante responde "debemos escribir la información y hacer un dibujo" (diario de observación, octubre de 2016).

\section{Conclusiones}

En el estudio encontramos que las maestras dan importancia a la memoria en la enseñanza y aprendizaje de las ciencias sociales. Para ellas, la memoria juega un papel relevante como huella del aprendizaje, como base para el desarrollo de pensamiento superior y, finalmente, como legado que permite dar significado a los hechos históricos. En este sentido, las docentes reconocen que los dispositivos de memoria, tales como el cuaderno de apuntes y la memoria iconográfica, son importantes en el acto de enseñar las ciencias sociales. Adicionalmente, consideran que se pueden desarrollar niveles de pensamiento superior, tales como, comparar, criticar, comprender, crear, relacionar y analizar, a partir del recordar. Así pues, los resultados de la prueba aplicada a los estudiantes demostraron que ellos alcanzaron el desarrollo de niveles de pensamiento como recordar y reproducir, sin embargo, no se evidencia que los jóvenes logren alcanzar los niveles de pensamiento superior.

La credibilidad y validez del estudio se cimentan en la rigurosidad del diseño y el manejo de los datos. La prueba diseñada y validada brindó resultados viables que facilitaron la interpretación de las categorías y la triangulación con las concepciones y las prácticas.

Por otra parte, consideramos que una de las principales limitaciones del estudio es el corto tiempo de ejecución. Esto impidió analizar si los estudiantes posteriormente alcanzan el nivel de pensamiento extendido, tal y como lo perciben las docentes. Adicionalmente, pensamos que existió desatención en algunos estudiantes en el momento de responder la prueba. Esto afectó potencialmente los resultados e impidió tener una visión más verídica de la realidad observable.

Finalmente, recomendamos ampliar el estudio a los demás niveles de educación básica y media, con el fin, de analizar si la memoria y el desarrollo del primer nivel de pensamiento tienen un papel importante en el desarrollo del nivel de pensamiento extendido en la enseñanza de las ciencias sociales a lo largo del bachillerato en estudiantes de una institución de carácter oficial en la ciudad de Bogotá.

Con este proyecto se cambia del paradigma tradicional de la evaluación sumativa a la comprensión de la evaluación formativa, donde el papel de la retroalimentación es importante para consolidar el aprendizaje de los estudiantes. Creemos que lo anterior es de vital importancia para nuestras prácticas pedagógicas, mejorando los procesos de enseñanza y evaluación dentro de cada uno de los contextos donde nos desempeñamos.

\section{REFERENCIAS}

Aguado, L. (2001). Aprendizaje y memoria. En Revista de Neurología, 32(4), pp. 373-381

Allen, J. (2012). Tipos de memoria: ¿cómo almacena los recuerdos el cerebro humano? Asociación de psicología Española Tarragona Barcelona. Recuperado en agosto de 2016. https://psicologiaymente.net/neurociencias/tipos-de-m emoria 
Atkinson, R.C. y Shiffrin, R.M. (1968). Human memory: a proposed system and its control processes. The psychology of learning and motivation: advances in research and theory, Vol. 2, pp. 89-195. New York: Academic press

Ballesteros, S. (1999). La memoria humana, investigación y teoría. Psicothema. Vol. 11, nº 4, pp. 705-723 ISSN 0214 - 9915 CODEN PSOTEG

Barron, C. (2015). Concepciones epistemológicas y práctica docente. Una revisión. En REDURevista de docencia universitaria, 13(1), pp. 35-56

Black, P., \& William, D. (1998). Inside the Black Box: Raising Standards Through Classroom Assessment. En: Revista Phi Delta Kappan, october, pp. 139-148

Denzin, N.K.y Lincoln, Y.S. (2000). The discipline and practice of qualitative research. En N. K. Denzin y Y.S. Lincoln (Eds.) Handbook of Qualitative Research. London: Sage Publications

Downing, S. (2006). Twelve Steps for Effective test Development. En: Handbook of test Development pp 3 - 25. London: Lawrence Erlbaum Associates

Duarte, J. Bos, M. y Moreno, J. (2012). Calidad, Igualdad y Equidad en la Educación Colombiana (Análisis de la prueba SABER 2009). BID. Recuperado de: http://idbdocs.iadb.org/wsdocs/getDocument.aspx?DOCNUM=36784 396

Feixas, M (2010). Enfoques y concepciones docentes en la universidad. En Revista RELIEVE, vol.16, n2, pp.1-27

Flick, U. (2002) Qualitative research designs. Designing qualitative research. London, Great Britain. Sage Publications

Freire, P. (1968). Pedagogía del oprimido. Montevideo: Siglo XXI Editores

Gómez, C. \& Miralles, P. (2015). ¿Pensar históricamente o memorizar el pasado? En: Revista de estudios sociales.n52, pp. $52-68$

Gutiérrez B. M. (2004). Investigación cualitativa aplicada a la Ciencia Política. Pontificia Universidad Javeriana. Bogotá- Colombia. Recuperado de http://www.javeriana.edu.co/blogs/mlgutierrez/files/Enfoques-y-estrategia s-de-investigacion4.pdf

Heller, A. (2001). Memoria cultural, identidad y sociedad civil. En Revista: Internationale Politik und Gesellschaft, 2, pp. 139-143

Herrera, P.A., Gámez, P.A., Torres, A., Corredor, J.C. \& Quintero, F. (2008). ¿Qué pensamos cuando evaluamos? La evaluación al tablero. En Revista Enunciación No. 13, 38-44. Bogotá D.C.: Universidad Distrital Francisco José de Caldas

Lepe, P. (2009). Enfoque histórico, hermenéutico y crítico, como modelo de investigación en la filosofía política. En Revista:Antroposmoderno. Recuperado de http://antroposmoderno.com/antro-articulo.php?id_articulo=1277

Li, M. \& Shavelson, R. (2003). Validating the Links between Knowledge and Test Items from a Protocol Analysis. College of Education, University of Washington

López, A. (2013). Estrategias de Evaluación. En: López, A. La evaluación como herramienta para el aprendizaje. Conceptos, estrategias y recomendaciones. Bogotá D.C.: Magisterio

Márquez, J. (1998). Panorama de los Programas de Habilidades de Pensamiento. Ponencia presentada en el Congreso de Psicoterapia y Desarrollo Infantil. Puebla: UDLA

McMillan, J. H. (2001). The role of assessment in teaching. Series Editorial Assistant: Shannon Morrow (Eds.). Classroom assessment, principles and practice for effective instruction. Boston: Allyn and Bacon

McMillan, J. H. (2007). Formative Classroom Assessment: The Key to improving student achievement. Educational Measurement: issues and practice. (pp. 1-7)

Ministerio de Educación Nacional (MEN) (2002) Lineamientos curriculares para el área de ciencias sociales. Serie lineamientos curriculares. Bogotá: Ministerio de Educación Nacional

Ministerio de Educación Nacional (MEN) (2004) Estándares Básicos de Ciencias Sociales. Bogotá: Ministerio de Educación Nacional

Montoya, J. (2015). El campo de los estudios curriculares en Colombia. Universidad de los Andes: Colombia

Nickerson, R.S., Perkins D. \& Smith E.E., (eds.) (1990). Enseñar a pensar: aspectos de la aptitud intelectual. Barcelona: Paidos 
Nitko \& Brookhart (2007). Assessament and grading in classroom. New Jersey: Person.

Ortega, C. y Franco, J. (2010). Neurofisiología del aprendizaje y la memoria. Plasticidad neuronal. En Revista Archivos de medicina. Recuperado de: http://www.archivosdemedicina.com/medicina-de-familia/neurofisiologa-del-apr endizaje-y-la-memoria-plasticidad-neuronal.pdf

Parga, M.H. (2002). El desarrollo de habilidades de pensamiento de orden superior como base metodológica para la realización de proyectos de investigación en diseño y para diseño. Memoria del Primer Seminario de Docencia del Diseño Industrial. Eds. Alfonso Zamora y Octavio García. México: UAM Azcapotzalco

Puentes, L.A. (2009) "Tensiones y distensiones en la práctica evaluativa”. Tesis de maestría no publicada. Pontificia Universidad Javeriana, Bogotá, Colombia

Reynolds, C., Livingston, R., \& Willson, V. (2006). The Initial Steps in Developing a Classroom Test. En Measurement and Assessment. United States of America: Pearson

Reynolds, C., Livingston, R., \& Willson, V. (2006). The Development and Use of selected-response Items. En Measurement and Assessment in Education. United States of America: Pearson

Rose, T. (2015). The end of average. How we succeed in a world that values sameness. New York: Haper one

Rueda, R. (2012). Sociedades de la información y el conocimiento: tecnicidad, phármokon e invención. En Revista: Nómadas 36. Universidad Central. Colombia

Santiago, J. (2005). Efectos de la memorización en la práctica escolar cotidiana de la enseñanza geográfica. Revista de Teoría y Didáctica de las Ciencias Sociales, enero-diciembre, 49-66

Stake, R.E. (2010). Qualitative Research. Nueva York: Guilford

Webb, N. L. (1997). Determining Alignment of Expectations and Assessments in Mathematics and Science Education. En Revista: NISE Brief 1(2), 2 